\title{
Wavelength-tunable picosecond pulses from a passively mode-locked figure-eight Erbium-doped fiber laser with a Sagnac fiber filter
}

\author{
B. Ibarra-Escamilla \\ baldemar@inaoep.mx \\ O. Pottiez \\ pottiez@cio.mx
}

\section{J. W. Haus}

Joseph.Haus@notes.udayton.edu

\section{E. A. Kuzin \\ ekuz@inaoep.}

\section{Bello-Jiménez}

\section{A. Flores-Rosas}

\author{
Electro-Optics Program, University of Dayton, Dayton OH 45469, USA \\ Instituto Nacional de Astrofísica, Optica y Electrónica, Departamento de Optica, Puebla, \\ Pue 72000, México \\ Centro de Investigaciones en Optica, León, Gto 37150, México
}

Electro-Optics Program, University of Dayton, Dayton OH 45469, USA

\begin{abstract}
Instituto Nacional de Astrofísica, Optica y Electrónica, Departamento de Optica, Puebla, Pue 72000, México

Instituto Nacional de Astrofísica, Optica y Electrónica, Departamento de Optica, Puebla, Pue 72000, México

Instituto Nacional de Astrofísica, Optica y Electrónica, Departamento de Optica, Puebla, Pue 72000, México
\end{abstract}

We experimentally demonstrated a wavelength-tunable passively mode-locked Erbium-doped figure-eight fiber laser. Wide tunability is achieved using a high-birefringence (hi-bi) fiber Sagnac loop. The filter transmission pass wavelength is controlled by thermally adjusting the hi-bi fiber loop length. The output pulses are wavelength tunable over a range from $1525 \mathrm{~nm}$ to $1555 \mathrm{~nm}$. The FWHM of the autocorrelation trace is about $3.1 \mathrm{ps}$ and the pulse spectrum has a FWHM of $1.5 \mathrm{~nm}$. The pulse temporal and spectral widths remain constant over the tunable range. [DOI: 10.2971/jeos.2008.08036]

Keywords: mode-locked fiber laser, nonlinear optical loop mirror, tunable lasers

\section{INTRODUCTION}

Wavelength-tunable sources are important for application such as fiber optics sensing, instrumentation, medicine, and optical communication. Recent papers considering a Sagnac fiber filter with high-birefringence (hi-bi) fiber in the loop have reported tunable wavelength operation [1]-[11]. All of these papers have demonstrated tunability for continuous wave (cw) operation, and some of them report multiple wavelength emission.

A passive mode-locked fiber laser is a simple and effective setup to generate ultrashort pulses. Many mode-locking techniques have been reported to implement a passive modelocked laser including semiconductor saturable absorber, nonlinear amplifier loop mirror (NALM), nonlinear optical loop mirror (NOLM) and nonlinear polarization rotation with a polarizer. Configurations that include a NOLM or a NALM are called figure-eight fiber lasers (F8L) [12]-[15]. The NOLM is usually formed by an asymmetrical coupler whose output ports are connected to form a loop [16]. The nonlinear phase shift of these beams is due to self phase modulation effect [17]. If a symmetrical coupler is used in the loop, the switching can be obtained through the dispersion effects or dependence of the nonlinear phase shift on polarization. In previous papers we reported the operation of a NOLM with a symmetrical coupler, a highly twisted fiber and a Quarter-Wave Retarder (QWR) in the loop to break the polarization symme- try of the counter-propagating beams [18]-[20]. For low input power the NOLM transmission is adjustable as well as the slope of the nonlinear dependence by a simple rotation of the QWR. High twist reduces the fiber residual linear birefringence and makes it immune to changes of environmental conditions. It was shown in [21], that twisted fiber operates as an ideal fiber without linear birefringence. A symmetrical NOLM with a QWR was developed as a mode-locker and investigated in a F8L design [22]-[24].

Pulses whose duration is few tens of picoseconds have not been widely reported in the literature. However the use of pulses whose duration is picoseconds or longer can be useful for many applications, for example even supercontinuum generation. The physical mechanisms of supercontinuum generation are quite distinct for femto- and picosecond pulses. To the best of our knowledge, no papers report on the generation of broadly wavelength-tunable picosecond pulses using a passively mode-locked F8L.

In this paper we experimentally demonstrate the wavelength tunability of a novel F8L by inserting a hi-bi fiber Sagnac loop into the cavity. Our mode-locked laser is based on a symmetrical NOLM with high-twist low-birefringence fiber and a QWR in the loop. The signal at the NOLM output is amplified by $1 \mathrm{~m}$ of Erbium-doped fiber. The output pulses are wavelength 
tunable over a range from $1525 \mathrm{~nm}$ to $1555 \mathrm{~nm}$. This tuning range is less than that demonstrated with other techniques using active elements and free space filters. The filter transmission function can be changed by selecting the hi-bi fiber loop length. The wavelength of the transmission maximum of the Sagnac loop is adjusted by changing the temperature of the hi-bi fiber, which in turn changes the optical path length of the fiber cavity. The FWHM of the pulse autocorrelation trace is about $3.1 \mathrm{ps}$ and the spectrum FWHM is $1.5 \mathrm{~nm}$. The pulse temporal and spectral widths remain constant over the tunable range.

\section{EXPERIMENTAL SETUP AND RESULTS}

Figure 1 is a sketch of the experimental setup. The NOLM is formed by a symmetrical Coupler 1, whose output ports were fusion-spliced with a $220 \mathrm{~m}$ loop of low-birefringence, highly twisted Corning SMF-28 fiber. A twist rate of 7 turns/m was imposed on the fiber in the NOLM loop. Prior to the NOLM input, we inserted the QWR2 to circularly polarize the clockwise (CW) beam. QWR1 was inserted in the loop to transform the counter-clockwise beam (CCW) from circular to linear polarization just after the Coupler 1. To form the QWR the coupler fiber arm is wrapped around a cylinder. Special attention was given to the proper choice for the cylinder diameter, which was experimentally determined to be $38 \mathrm{~mm}$. To calibrate the retarder we introduced circularly polarized light and measured the ellipticity at the output. We found that the transmitted power extinction ratio $P_{\min } / P_{\max }$ was always less than $5 \times 10^{-3}$. A $980 \mathrm{~nm}$ pump laser with a $100 \mathrm{~mW}$ maximum power was used. Pump power was injected into the amplifier through a WDM coupler. The signal at the NOLM output was amplified by $1 \mathrm{~m}$ of Erbium-doped fiber (with an Erbium concentration of $9.92 \times 10^{24} \mathrm{~m}^{-3}$ ) and passed through a polarization dependent circulator (C1), which served as polarizer and isolator. The polarization controller (PC1) was adjusted for maximal transmission through $\mathrm{C} 1$. The QWR2 transforms

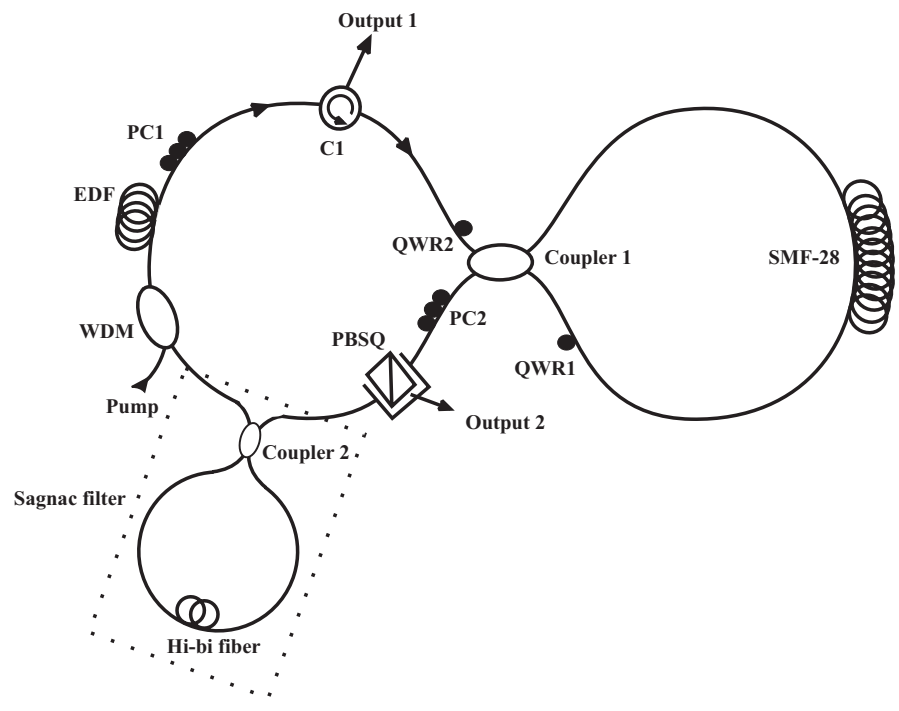

FIG. 1 Experimental setup with a Sagnac filter at the NOLM output. EDF $=1 \mathrm{~m}$ $(15.35 \mathrm{~dB} / \mathrm{m}$ of peak absorption near $1530 \mathrm{~nm})$. The Sagnac filter (inside the dotted rectangle) central wavelength is adjusted by changing the fiber temperature with a TEC. linear polarization to circular polarization at the NOLM input. We used a polarizer beam-splitter cube (PBSQ) at the NOLM output to adjust the best quality of output pulses from the laser. The PC2 is adjusted to have minimal signal at the laser Output 2 in cw operation. This means at the same time that we adjusted the maximum transmission through the PBSQ to the circulator C1. In the linear regime the NOLM operates as a half wave plate and therefore the output polarization is orthogonal to the input one. So with this adjustment the orthogonal polarization component at the NOLM output passes through the PBSQ and the parallel polarization component is monitored at Output 2. The parallel component is zero for low input power; it is nonzero only at high power.

The hi-bi Sagnac fiber filter in Figure 1 is formed by a symmetrical Coupler 2 and a $12 \mathrm{~cm}$ segment of a hi-bi fiber (Fibercore F-SPS with a beat length $<1.5 \mathrm{~mm}$ ). The detailed description of the Sagnac filter used here can be found in [25]. The Sagnac fiber filter provides a periodic transmission that can be used as a band-pass filter by carefully selecting the hi-bi fiber length. For the laser configuration, we chose $12 \mathrm{~cm}$ of a hi-bi fiber, which provides a half period of the transmission dependence on the wavelength equal to $15 \mathrm{~nm}$ that allows only one transmission maximum in the range between $1525 \mathrm{~nm}$ and $1555 \mathrm{~nm}$. The maximal transmission wavelength is adjusted by changing the fiber's temperature using a thermo-electric cooler (TEC). Figure 2 shows five experimental spectra measured at Output 2 with different TEC settings for cw operation of the laser. With this system, we can shift the wavelength in the range from $1525 \mathrm{~nm}$ to $1555 \mathrm{~nm}$; the rate of wavelength shift with temperature is $1 \mathrm{~nm} /{ }^{\circ} \mathrm{C}$.

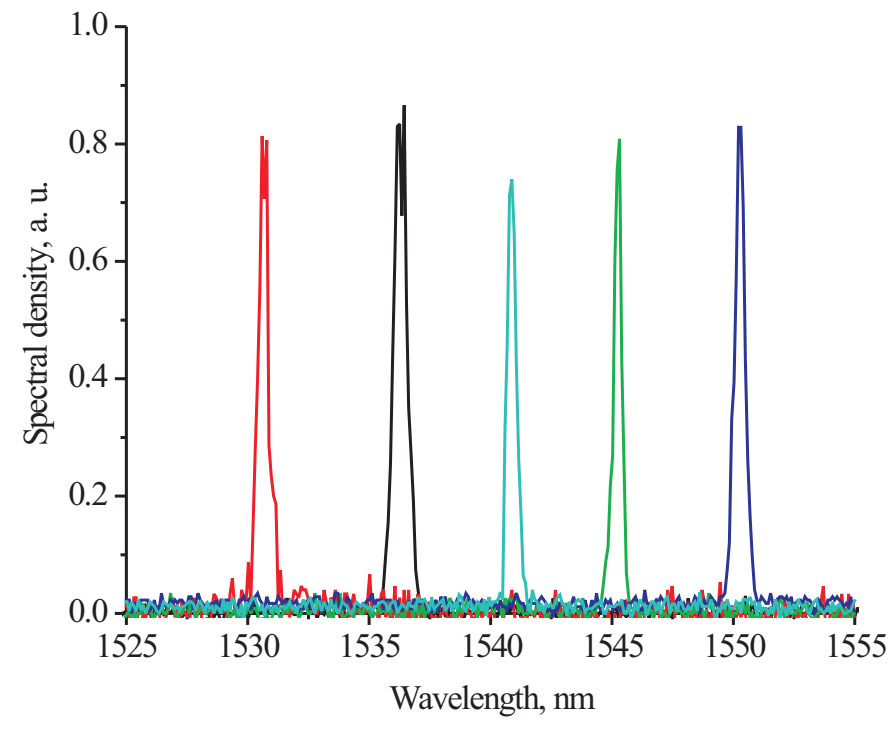

FIG. 2 Experimental continuous wave spectra from the laser at Output 2. Within experimental uncertainty the widths of the spectra are the same across the entire range.

In a previous paper we reported conditions for self-starting operation of a F8L based on a power-balanced NOLM with a twisted low-birefringence fiber and a QWR in the loop $[23,24]$. Self-starting passive mode-locking operation of a F8L can be obtained through a simple and repeatable adjustment of NOLM by using the QWR1. The rotation of the QWR1 adjusts desirable low power transmission and nonlinearity. Selfstarting mode-locking appear at specific and always the same 
low-power transmission of the NOLM. In this new configuration, mode-locked pulses are generated by adjusting the QWR1 (in Figure 1) to the orientation for which we get selfstarting in $[23,24]$, but in this case we need to apply an external stimulation, these pulses are generated for pump power higher than $50 \mathrm{~mW}$. Above this power multiple pulses coexist in the laser cavity. The number of pulses in the cavity is reduced to one by decreasing the pump power to $15 \mathrm{~mW}$. At this pump power only one high quality pulse circulates in the cavity. The repetition period of the pulses is $1.15 \mu$ s which corresponds to a $0.87 \mathrm{MHz}$ repetition frequency.

Figure 3 shows five experimental spectra of mode-locked operation measured at Output 2 with five different values of TEC current. In this figure, the FWHM bandwidth is the same at each one of the wavelengths, and is equal to $1.5 \mathrm{~nm}$. We note that once mode-locking operation is achieved for a given wavelength, then the cavity remains modelocked as the current of the TEC is changed to tune the pulse wavelength.

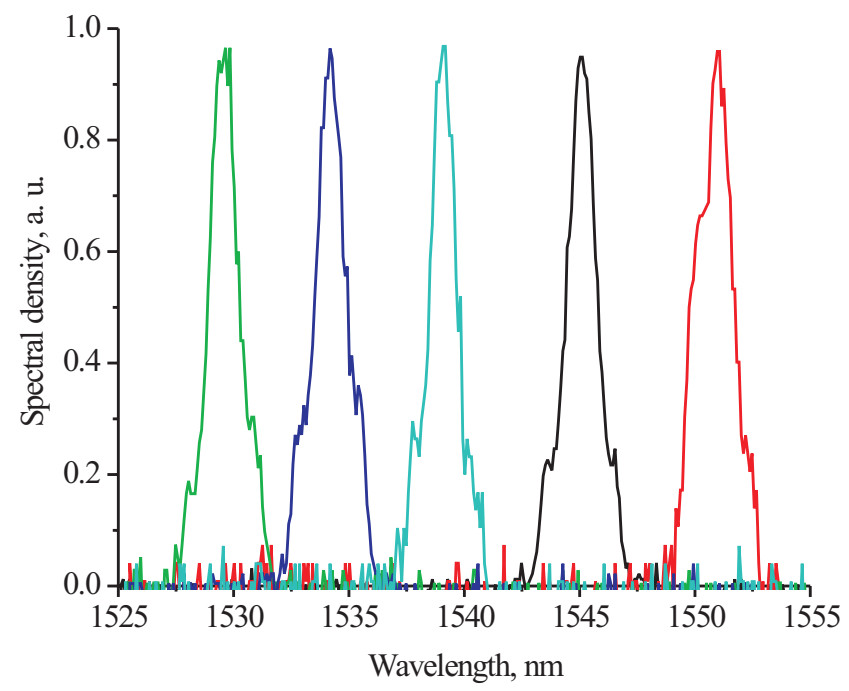

FIG. 3 Experimental mode-locked spectra from the laser at Output 2. Within experimental uncertainty the widths of the spectra are the same across the entire range.

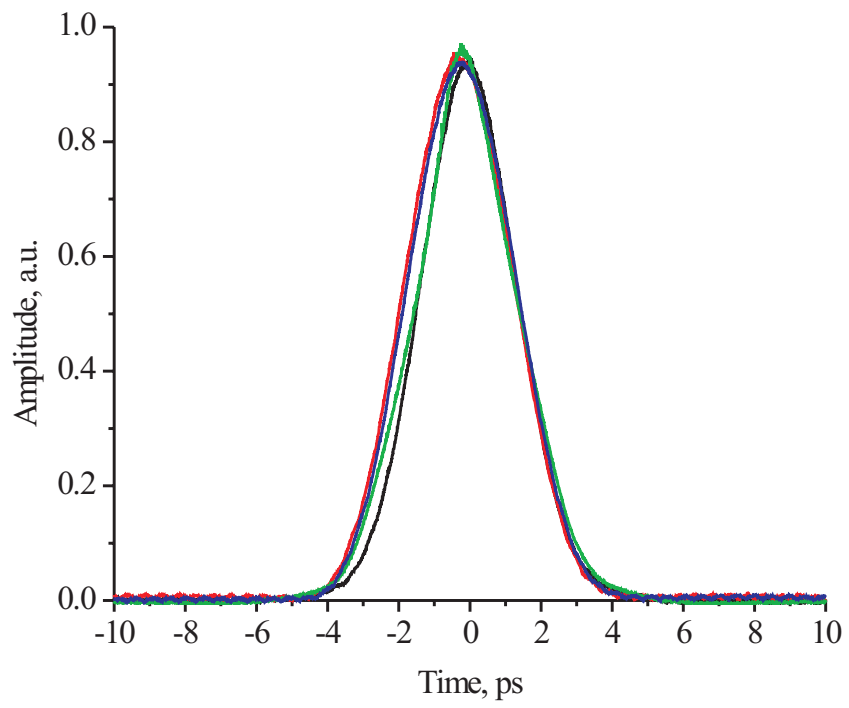

FIG. 4 Autocorrelation trace measured at Output 2. The autocorrelations are plotted for different wavelengths and the results are practically indistinguishable from one another.
Figure 4 shows the autocorrelation traces measured at Output 2 for different wavelengths. We found that the curves are nearly the same for all wavelengths. The FWHM of the autocorrelation trace is about $3.1 \mathrm{ps}$ in all cases, corresponding to a FWHM pulse duration $\Delta \tau=2.2 \mathrm{ps}$, if a Gaussian profile is assumed.

The FWHM bandwidth of the output pulse spectrum measured at Output 2 is $\Delta \lambda=1.5 \mathrm{~nm}$, which corresponds to the time-bandwidth product $\Delta v \Delta \tau=0.418$, where $\Delta v$ is the FWHM of the frequency spectrum. For a Gaussian profile the Fourier transform limit is 0.441 , which is slightly larger and suggests a pulse that is more complex than a Gaussian.

\section{CONCLUSIONS}

We demonstrate the operation of a broadly wavelengthtunable passive figure-eight fiber laser including a Sagnac fiber filter with high-birefringence (hi-bi) fiber loop into the cavity. The signal at the NOLM output was amplified by $1 \mathrm{~m}$ of Erbium-doped fiber. The output pulses are wavelengthtunable over a range from $1525 \mathrm{~nm}$ to $1555 \mathrm{~nm}$ by inserting a Sagnac fiber filter at the NOLM output. The maximal transmission wavelength is adjusted by changing the temperature of the hi-bi fiber. Mode-locked laser operation was achieved at a specific position of the QWR for pump powers higher than $50 \mathrm{~mW}$ yielding multiple pulses in the laser cavity. The pump power is reduced to $15 \mathrm{~mW}$ so that only one high quality pulse remains in the cavity. The FWHM of the autocorrelation trace is about 3.1 ps and the pulse spectrum has a FWHM of $1.5 \mathrm{~nm}$. The pulse temporal and spectral widths remain constant over the tunable range.

\section{ACKNOWLEDGEMENTS}

BIE was supported by CONACYT grant 80451 for a Sabbatical year at the University of Dayton. EK was supported by CONACYT grant 47169.

\section{References}

[1] X. Shu, S. Jiang, and D. Huang, "Fiber grating Sagnac Loop and its multiwavelength-laser application" IEEE Photonic Tech. L. 12, 980-982 (2000).

[2] N. J. C. Libatique, and R. K. Jain, "A broadly tunable wavelengthselectable WDM source using a fiber Sagnac loop filter" IEEE Photonic Tech. L. 13, 1283-1285 (2001).

[3] Y. W. Lee, K. J. Han, B. Lee, and J. Jung, "Polarization-independent all-fiber multiwavelength-switchable filter based on a polarizationdiversity loop configuration" Opt. Express 11, 3359-3364 (2003).

[4] K. L. Lee, M. P. Fok, S. M. Wan, and C. Shu, “Optically controlled Sagnac Loop comb filter" Opt. Express 12, 6335-6340 (2004).

[5] C. Das, and J. W. Y. Lit, "Wavelength switching of a fiber laser with a Sagnac loop reflector" IEEE Photonic Tech. L. 16, 60-62 (2004).

[6] Y. W. Lee, J. Jung, and B. Lee, "Multiwavelength-switchable SOAfiber ring laser based on polarization-maintaining fiber loop mirror and polarization beam splitter" IEEE Photonic Tech. L. 16, 54-56 (2004). 
[7] Y. W. Lee, and H. T. Kim, "Wavelength-switchable flat-top fiber comb filter based on a Solc type birefringence combination" opt. Express 13, 1039-1048 (2005).

[8] J. Huixtlaca-Cuatecatl, G. Beltrán-Pérez, J. Castillo-Mixcóatl, and S. Muñoz-Aguirre, "Fiber Laser Thermally Tunable by a Filter Composed of Two Sagnac Interferometers" IEEE J. Quantum Elect. 44, 49-55 (2008).

[9] Z. Zhang, J. Wu, K. Xu, X. Hong, and J. Lin, "Polarization-dependent output states of a fiber laser with nonlinear polarization rotation" Opt. Eng. 47, 085002 (2008).

[10] F. W. Sheu, C. Y. Chiou, and S. C. Yang, "Performance of a wavelength-tunable erbium-doped fiber laser using a Sagnac interferometer" Opt. Commun. 281, 4719-4722 (2008).

[11] M. A. Mirza, and G. Stewart, "Theory and design of a simple tuneable Sagnac loop filter for multi-wavelength fibre lasers" Appl. 0pt. to be published (2009).

[12] I. N. Duling III, "All-fiber soliton laser mode locked with a nonlinear mirror" Opt. Lett. 16, 539-541 (1991).

[13] A. G. Bulushev, E. M. Dianov, and 0. G. Okhotnikov, "Self-starting mode-locked laser with a nonlinear ring resonator" Opt. Lett. 16, 88-90 (1991).

[14] I. N. Duling III, and M. L. Dennis, Compact Sources of Ultrashort Pulses (Cambridge University Press, Cambridge, 1995).

[15] Z. X. Zhang, Z. Q. Ye, M. H. Sang, and Y. Y. Nie, "Passively modelocked fiber laser based on a symmetrical nonlinear optical loop mirror" Laser Phys. Lett. 5, 364-366 (2008).

[16] N. J. Doran, and D. Wood, "Nonlinear-optical loop mirror" Opt. Lett. 13, 56-58 (1988).

[17] G. P. Agrawal, Applications of Nonlinear Fiber Optics (Academic Press, San Diego, 2001).
[18] E. A. Kuzin, J. A. Andrarde-Lucio, B. Ibarra-Escamilla, R. RojasLaguna, and J. Sanchez-Mondragon, "Nonlinear optical loop mirror using the nonlinear polarization rotation effect" Opt. Commun. 144, 60-64 (1997).

[19] 0. Pottiez, E. A. Kuzin, B. Ibarra-Escamilla, and F. MendezMartinez, "Easily tunable nonlinear optical loop mirror including low-birefringence, highly twisted fibre with invariant output polarization" Opt. Commun. 229, 147-159 (2004).

[20] B. Ibarra-Escamilla, E. A. Kuzin, P. Zaca-Morán, R. Grajales-Coutiño, F. Mendez-Martinez, 0. Pottiez, R. Rojas-Laguna, and J. W. Haus, "Experimental investigation of the nonlinear optical loop mirror with twisted fiber and birefringence bias" Opt. Express 13, 1076010767 (2005).

[21] T. Tanemura, and K. Kikuchi, "Circular-birefringence fiber for nonlinear optical signal processing" J. Lightwave Technol. 24, 41084119 (2006).

[22] E. A. Kuzin, B. Ibarra-Escamilla, D. E. García-Gómez, and J. W. Haus, "Fiber laser modelocked by the nonlinear polarization rotation Sagnac interferometer" Opt. Lett. 26, 1559-1561 (2001).

[23] B. Ibarra-Escamilla, 0. Pottiez, E. A. Kuzin, R. Grajales-Coutiño, and J. W. Haus, "Experimental investigation of a passively mode-locked fiber laser based on a symmetrical NOLM with highly twisted lowbirefringence fiber" Laser Phys. 18, 914-919 (2008).

[24] B. Ibarra-Escamilla, 0. Pottiez, E. A. Kuzin, J. W. Haus, R. GrajalesCoutiño, and P. Zaca-Moran, "Experimental investigation of selfstarting operation in a F8L based on a symmetrical NOLM" Opt. Commun. 281, 1226-1232 (2008).

[25] M. Bello-Jiménez, E. A. Kuzin, B. Ibarra-Escamilla, and A. FloresRosas, "Optimization of the two-stage single-pump Er-doped fiber amplifier with high amplification for low frequency ns-scale pulses" Opt. Eng. 46, 125007 (2007). 